\title{
Vaccine Adjuvants: The Current Necessity of Life
}

\author{
Amit Gupta ${ }^{1, *}$; Sushama R. Chaphalkar ${ }^{1}$

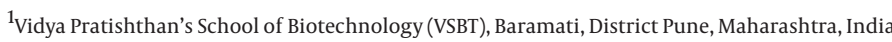 \\ ${ }^{*}$ Corresponding Author: Amit Gupta, Department of Immunology, VSBT (Research centre affiliated to Savitribai Phule Pune University) Baramati, Maharashtra, INDIA. Tel: +91 \\ 2112239386, E-mail:amitvsbt@gmail.com; amitgupta@vsbt.res.in
}

Received: February 18, 2015; Accepted: June 28, 2015

\begin{abstract}
Context: Vaccination is considered as the most cost effective method for the prevention of human diseases. For this prevention method, we need certain substances to increase or boost the antibody as well as cell-mediated immune response against various bacterial as well as viral pathogens. Until now, alum was considered as the safest adjuvant for human use, licensed by the United States Food and Drug Administration. Due to the poor adjuvanticity of alum, conventional vaccines require multiple recall injections, at different time intervals, to attain or sustain the optimal immune response. The present review discusses about the necessity of adjuvants for vaccines.

Evidence Acquisition: A number of factors such as slow release of antigen (depot effect), more efficient delivery of antigen to draining lymph nodes, non-specific activation of antigen-presenting cells or of B and/or T-lymphocytes, increased uptake of antigen by antigenpresenting cells or increased recruitment of immune cells to the site where the antigen is present, can contribute to increased immune responses to immunization. Many of these factors involve the interaction of various immune system components and specific anatomical features, making them difficult to replicate in model systems in vitro.

Results: Despite the development of many potent adjuvant formulations with vaccine antigen during the last 80 - 90 years, aluminum compounds are still the only approved adjuvants used for routine human vaccines.

Conclusions: Based on pre-clinical and preliminary clinical observations, it appears that the range of adjuvants accepted for human vaccines will expand in the coming years.
\end{abstract}

Keywords: Vaccination; Adjuvant; Alum

\section{Context}

The goal of vaccination is to generate a strong immune response providing long term protection against infection. Unlike attenuated live vaccines, killed whole organisms or subunit vaccines, generally require the addition of an adjuvant to be effective. Adjuvants are compounds that enhance the immune response against co-inoculated antigens with the word adjuvant coming from the Latin word adjuvare (to help or to enhance). Adjuvants have been used to improve vaccine efficacy from the early 1920's (1, 2). In 1926, Glenny demonstrated the adjuvant activity of aluminum compounds (1). More than ten years later, in the late 1930s, Freund developed his water-in-oil/ oil-in-water emulsion containing killed mycobacteria (3). Complete Freund's adjuvant containing the killed form of mycobacterium is still one of the most well known powerful immunologic adjuvants, yet it is too reactive to be used clinically. However, incomplete Freund's adjuvant (waterin-oil emulsion, without mycobacterium) has been used in several vaccine formulations including an influenza vaccine formerly licensed in the United Kingdom, which has been administered to over one million people. In 1956, Johnson demonstrated the adjuvant activity of lipopolysaccharide endotoxins from gram-negative bacteria (4).
In 1974, Lederer and his collaborators identified muramyl dipeptide as the smallest active adjuvant component of mycobacteria (5). More than 300 synthetic derivatives of muramyl dipeptide have been produced in the search for molecules that retain their adjuvant activity yet in newborns (6), aged (7) and immunocompromised hosts (8). Adjuvants can also promote T-cell proliferation and cellmediated immunity (9). They can stimulate major histocompatability complex (MHC) class-I restricted CD8 + Cytotoxic T-Lymphocyte (CTL) responses when used with vaccine antigens that cannot achieve this alone or when formulated with alum. While the number of substances with adjuvant activity and describing their use has expanded enormously (10). Certain particulate adjuvants, such as Immunostimulating Complexes (ISCOMS), liposomes, and biodegradable microspheres can induce antibody responses at mucosal surfaces $(11,12)$.

\subsection{Characteristics of an Ideal Adjuvant}

Safety of adjuvant formulations is the biggest concern, particularly for routine childhood vaccines. Edelman (1980) (13) listed a number of criteria to ensure the safety of adjuvanted vaccines. In addition to safety with regards

Copyright (C) 2015, Shiraz University of Medical Sciences. This is an open-access article distributed under the terms of the Creative Commons Attribution-NonCommercial 4.0 International License (http://creativecommons.org/licenses/by-nc/4.0/) which permits copy and redistribute the material just in noncommercial usages, provided the original work is properly cited. 
to local reactions, systemic reactions (general toxicity and pyrogenicity), autoimmune diseases, hypersensitivity reactions, carcinogenicity, teratogenicity etc., an ideal adjuvant would be chemically defined so that it can be manufactured consistently. The preparation would elicit a protective immune response with weak antigens including polysaccharide-protein conjugates with lower doses of antigens and fewer injections. The adjuvant should be effective on infants and young children, ideally at birth and elicit a more persistent response of high quality (high affinity antibodies or desired type of IgG isotype). The adjuvant should be stable with regard to adjuvanticity and toxicity, without any interaction with the antigen. It should be biodegradable and non-immunogenic by itself. None of the adjuvants available at present meet these criteria.

\subsection{Types and Classification of Adjuvants}

Development of newer vaccines include highly purified subunit antigens that are weakly immunogenic in general. Vaccine formulations often require adjuvants for increased immunological efficiency and better vaccination schedules (14). Currently used adjuvants include aluminum hydroxide, aluminum phosphate, calcium phosphate, water-in-oil emulsions, products from bacteria and liposomes. Often there is compromise on the level of adjuvanticity and acceptable level of safety. Other adjuvants such as monophosphoryl lipid A, ISCOMS, QS-21 a purified saponin from bark of Quillaja saponaria Molina, and Syntex Adjuvant Formulation (SAF) are being investigated for development of better and safer adjuvants (14). Plant based immunomodulators are being considered as one option (15-17). Previously we have reported various immunomodulators of Ayurvedic origin. The extracts and formulations prepared from Ayurvedic medicinal plants including Withania somnifera, Emblica officinalis, Panax notoginseng, Tinospora cordifolia and Asparagus racemosus demonstrated significant immunostimulatory activity, particularly at humoral level in experimental systems with or without induced immunosuppression (1821). Attempts to organize adjuvants in grouped categories in order to facilitate adjuvant selection have sometimes been difficult because of multiple and overlapping biological effects of many adjuvants. However, this immunopharmacological approach can be useful for providing a prospective on the types of immunostimulators that are available. A practical categorization of different types of immunostimulators was proposed by Edelman during the 1980s (13). In this latter analysis, three general types of immunostimulatory compounds were proposed; adjuvants per se, carriers and vehicles.

1) Adjuvants per se- included in the adjuvant category are aluminum salts, saponin, muramyl di- and tripeptides, monophosphoryl lipid A, Bordetella pertussis, cytokines and many others. 2) Carriers, which mainly provide $\mathrm{T}$ cell help, include bacterial toxoids, fatty acids and living vectors. 3) Vehicle category includes mineral oil emulsions (e.g. incomplete Freund's adjuvant), biodegradable oil emulsions (e.g. emulsions containing peanut oil, squalene, or squalane), non-ionic block copolymer surfactants, liposomes, and biodegradable polymer microspheres.

\subsection{Parameters of Body Immune System Influenced By Adjuvants}

These adjuvants have the capability to influence or control many parameters of immune responses. The following have been demonstrated by ourselves and/or others: antibody production persistence, specificity, titer, duration, memory, class, isotype, avidity, cell-mediated immunity, generation of CD4 mediated mediated cell mediated immunity (CMI) delayed type hypersensitivity (DTH), and and generation of CD8 mediated cell mediated immunity (cytotoxic T lymphocyte), mucosal immunization and incidence of genetic non-responder.

It seems likely that adjuvants will be increasingly important as the science of vaccines advances. They could be improved to provide longer lasting protection with fewer inoculations. New vaccines are urgently needed for many infections including hepatitis B, HIV, malaria, tuberculosis etc. In developing vaccines for these infections, it is necessary to consider adjuvants as sophisticated agents or weapons, which can critically influence many parameters of immune responses including specificity, type, intensity and duration. The study of vaccine adjuvants is, in reality studying the control of the expression of different types of immune responses. Like other areas of medicine, it benefits greatly from advances in molecular biology and is an exciting area of developing science.

\subsection{Modes of Action of Adjuvants}

Adjuvants may act in one or more of the five mentioned ways.

\subsubsection{Immunomodulation}

This refers to the ability of many adjuvants to modify the cytokine network. In general, only immunomodulatory compounds will exert an adjuvant effect when presented at a separate time or site to the immunogen. Immunomodulation may result in a general up-regulation of the entire immune system, yet most commonly results in upregulation of certain cytokines and a concomitant down regulation of others. Two major subsets of $\mathrm{CD} 4+\mathrm{T}$ cells, viz Thl and Th2, have been well described for mice $(22,23)$ and humans $(24)$, and their existence are postulated for other animal species. Thl responses typically induce complement fixing antibody and strong Delayed Type Hypersensitivity (DTH) reactions, and are associated with IFN- $\gamma$, IL-2 and IL-12; whilst Th2 responses result in high circulating and secretory antibody levels, frequently IgE and cytokines IL-4, IL-5 and IL-6, and possibly IL-10. 
Thl and Th2 responses are mutually inhibitory (23).

Selection of the appropriate immunomodulatory adjuvant will not only lead to an enhanced immune response but will also determine the isotype of $\operatorname{IgG}$, which other immunoglobulins and how much CD4 + directed, cell mediated immunity (CMI) is generated. The immune response never deviates or swings totally in one direction (cellular or humoral). The most important adjuvants, which are approved for human use, are aluminum salts which induce Th2 response (24) and bacterial endotoxins and derivatives (lipid A, monophosphoryl lipid A), which induce a predominantly Thl type response (24). A good adjuvant may enhance a balanced immune response.
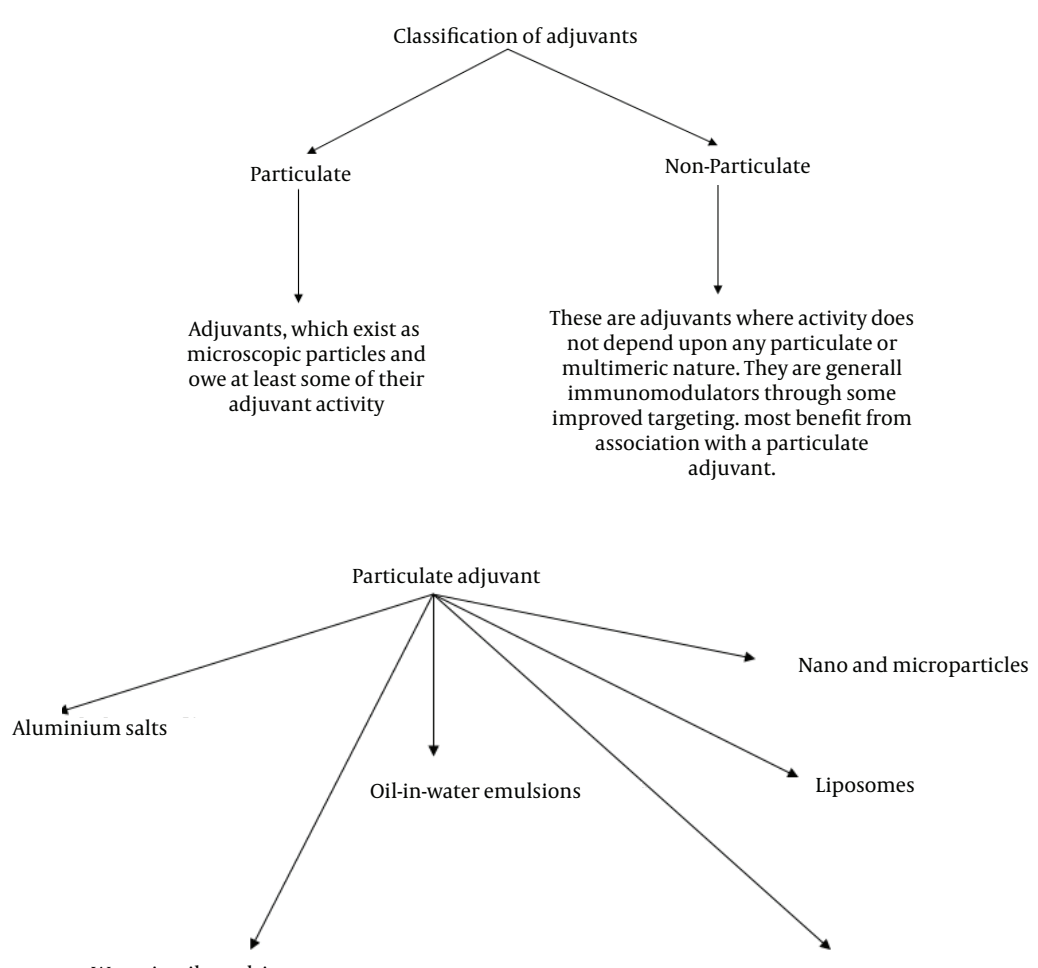

Water-in-oil emulsions

Immune stimulating complexes (ISCOM adjuvant-Iscoter $\mathrm{AB}$ )

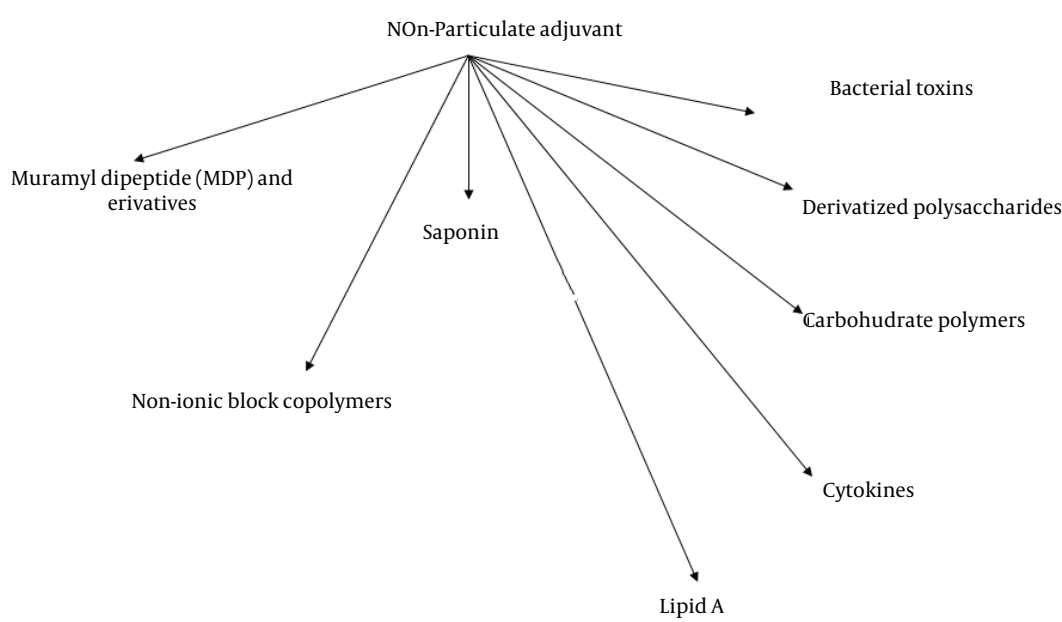

Figure 1. Classification of Adjuvants (i.e. Particulate and Non-Particulate) 

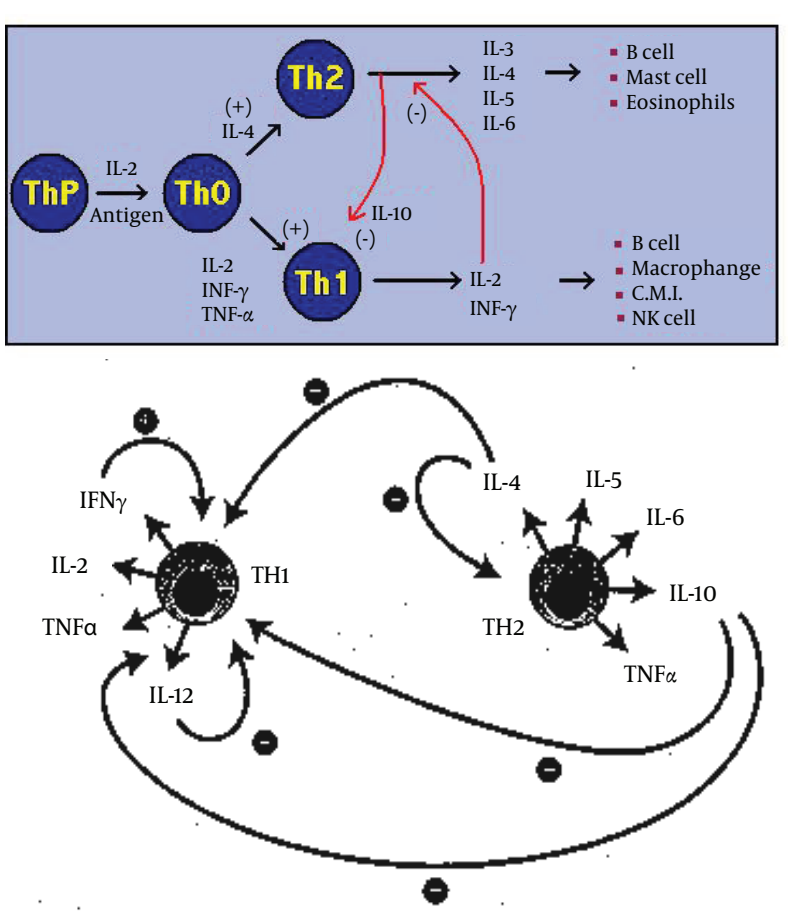

Figure 2. T helper (Th) Cells Associated With Cytokines Production

\subsubsection{Presentation}

This refers to the ability of an adjuvant to preserve the conformational integrity of an antigen and to present this to appropriate immune effector cells. This will occur when an adjuvant is able to interact with an antigen in such a way that conformational epitopes are more effectively maintained. The main benefits are an improved in vivo activity and an increased shelf life. Three major sets of interactions are required to achieve an effective antibody response.

1) The first interaction is with professional antigen presenting cells (APC), typically Dendritic cells (DC), Langerhans cells (LC) and possibly macrophages, although their role is still in dispute (25). Antigen is taken up by receptor-mediated endocytosis or fluid-phase pinocytosis (26) and the resultant endosome fuses with a lysosome to form an endolysosome. About this stage, external signals, probably dominated by granulocyte monocyte colony stimulating factor (GM-CSF), direct the DC to a regional draining lymph node or the spleen and initiate antigen processing and presentation. Antigen is processed into small peptides, which then meet with major histocompatibility class II molecules (MHC-II), which have already been assembled in the endoplasmic reticulum and are processed through the Golgi and trans-Golgi reticulum. The resultant complex is then transported to the surface of the APC, where the peptide is displayed in association with MHC-II (27). The APC will also secrete IL-l, the amount of which will be determined by the degree of upregulation of APC. This upregulation may be one of the functions of an immunomodulatory adjuvant. Local concentrations of IL-l will attract CD4 + cells to the APC, and those with T-Cell receptors (TCR) complementary to the peptide-MHC-II complex will undergo clonal expansion. It is probable that the Thl/Th2 switch is determined at this stage. If this is so, then an immunomodulatory adjuvant must be present, at this time, in an effective concentration.

2) The second interaction involves antigen and B cell, and recognition is primarily between surface immunoglobulin (28) and antigen. Bound antigen is internalized by Ig receptor-mediated endocytosis, digested in an endolysosome, and the resultant peptides are again expressed on the B cell surface in association with MHCII. By this time, T cell subsets capable of recognizing this complex have already undergone clonal expansion and are available to help and direct the B cell to clonally expand into plasma cells, which actively excrete immunoglobulin of the same specificity as the Ig on the surface of the initial B cell. Cytokine exchange will also determine the antibody isotype.

3) The third interaction is partly speculative. Although there is increasing supportive evidence (29) that follicular dendritic cells can provide a long-term reservoir of native antigen, which is essential both for effective affinity maturation of the immune response and for persistence of biologically relevant antibody production, it is argued that these responses depend upon availability of antigen in native conformation. Thus, antigen presentation confers three major benefits; firstly it will maximize the amount of conformationally relevant (i.e. neutralizing) antibodies, secondly it will influence the affinity of the antibody and finally it can influence the duration of the immune response.

\subsubsection{Induction of CD8 + Cytotoxic T-Lymphocyte (CTL) Responses}

The induction of CTL responses generally requires antigen to be processed within the cell cytosol (the endogenous pathway) where peptides, generally of nine amino acids 9 mers, become incorporated within the closed end groove of the MHC class I molecule and are then expressed on the cell surface. Current evidence suggests that turnover of cellular proteins occurs in a $26 \mathrm{~s}$ multi-enzyme complex. The proteolytic component of this complex is the proteasome, a highly conserved $20 \mathrm{~s}$ structure comprised of 24 - 28 subunits (30). The majority of proteins, which pass through this complex exit as peptides, which are further processed by exopeptidases to amino acids. However, a small proportion is selectively transported to the endoplasmic reticulum, by specialized transporter proteins (TAP1 and TAP2), where they are incorporated into the groove of the MHC-I and passed via the Golgi to the cell surface (27). There is increasing evidence that a low molecular mass protein (LMP) is a 
Gupta A et al.

specialized proteasome where two (or more) of the subunits are MHC encoded (27). The presence of these MHC encoded subunits within a proteasome may modify the proteolytic cleavage towards peptides, which are MHCcompatible. Production of LMP is upregulated by IFN- $\gamma$ (27), and it is tempting to speculate whether this is a mechanism by which the proportion of peptide capable of insertion into MHC-1 can be increased in response to a cytokine warning signal.

For an adjuvant to be useful for CTL induction, it must facilitate incorporation or persistence of appropriate peptides into MHC-1. The most effective way to achieve this is for the adjuvant to interact in some way with cell membranes so that the antigen associated with the adjuvant is deposited within the cytosol in a form suitable for normal processing in the proteasome. This may occur by fusion with the external membrane or by endocytosis/ pinocytosis followed by endosome membrane fusion or rupture (endosomal escape). Incorporation of an immunomodulator within this adjuvant formulation, especially one that induces IFN- $\gamma$ production, could be expected to increase relevant MHC-1-peptide expression. Although most cells express MHC-1, the most effective target cell for CTL induction is an APC and most probably a DC.

\subsubsection{Targeting}

This defines the ability of an adjuvant to deliver an immunogen to immune effector cells, generally via APCs. Although little data exists, it is likely that the vast majority of the delivered vaccine is lost either by serum protease degradation or by first-pass removal in the liver. This form of adjuvant activity may not modify the type of immune response but rather will affect the amount of immunogen required to achieve a given effect i.e. the efficiency of the generation of the immune response. However, if targeting can be selective for macrophages rather than DC, or the converse, the type of immune response may be substantially modified (25) where depletion of macrophages lead to a strong Th2 shift in response. There are several ways in which an adjuvant can achieve this effect. The most common is to interact with antigen in a way to form multimolecular aggregates. These aggregates will encourage uptake by macrophages and DC, and if an immunomodulatory adjuvant is included, will ensure that the antigen and immunomodulator are delivered to the same APC.

Alternatively, delivery to macrophages (31) and DCs (32) can be increased when the adjuvant has sugar moieties (e.g. saponins) or other cell-surface-receptor recognizing molecules (e.g. recognition of GM-l ganglioside by Escherichia coli heat-labile enterotoxin (LTB) and and cholera toxin B (CTB) (33), or when immunogen can be attached to a mannose polymer (e.g. mannan and acemannan) (34) or other carbohydrate. A third option is for an adjuvant to saturate Kupffer cells in the liver so that antigen, preferably not connected to the adjuvant, can be preferentially taken up by APC.

\subsubsection{Depot Generation}

This can be achieved as a short or long term depot, the latter giving either a continuous or pulsed release. Short term depots are typified by aluminum salts and w/o emulsions, where antigen is trapped at the injection site and therefore cannot be lost by liver clearance. Excision of the injection site eight to ten days after dosing has little if any effect on magnitude or duration of response (3), suggesting that antigen has either been removed or walled-off by that stage. Long-term depots are best achieved using synthetic polymers such as polylactide coglycolide (PLG), to produce microspheres, which degrade to yield a pulsed delivery. These microspheres are preferably $>10 \mu \mathrm{m}$ in size, so that they remain at the injection site until biodegradation permits removal of their content (immunogen and preferably adjuvant) by APC. Release times from one to six months can be achieved with reasonable precision (29).

\section{Evidence Acquisition}

\subsection{Challenges of Adjuvant Research}

A number of factors such as slow release of antigen (depot effect), more efficient delivery of antigen to draining lymph nodes, non-specific activation of antigen-presenting cells or of B and/or T-lymphocytes, increased uptake of antigen by antigen-presenting cells or increased recruitment of immune cells to the site where the antigen is present, can contribute to increased immune responses to immunization. Many of these factors involve the interaction of various immune system components and specific anatomical features, making them difficult to replicate in model systems in vitro. Furthermore, the overall effect of an adjuvant may result from the interaction of multiple factors. For example, an oil-water emulsion (35) might provide more effective delivery of antigens to draining lymph nodes, increased uptake by antigen presenting cells and activation of antigen presenting cells or T cells, all of which may contribute to its effect. Thus the ability to develop mechanism-based in vitro screens for novel adjuvant compounds is severely limited at present. Most adjuvants have been identified on the basis of testing in vivo. Species differences in responsiveness also may complicate the search for novel adjuvants. Not all adjuvants that are active in mice, for example, are equally active in nonhuman primates or in humans.

\subsection{The Need for New Adjuvants}

Conventional vaccines based on either inactivated pathogens, live attenuated pathogens and more recently, subcellular components such as purified capsular polysaccharides isolated from bacteria, virus proteins or recombinant proteins, have been successfully used to induce neutralizing antibodies against surface molecules in order to prevent infection. To achieve this aim, adjuvant preparations containing alum have been used 
to adsorb bacterial or viral antigens (36). In such formulations both electrostatic and hydrophobic interactions were shown to contribute to the adsorption of antigens by aluminum-based adjuvants (37). Expectations for new vaccines have changed dramatically in the recent years. Vaccines based on live attenuated or inactivated pathogens might still have a role to play in the future of vaccination. However, in the context of a tougher regulatory environment, most experimental vaccines which are currently being tested on humans are based on recombinant viruses, recombinant proteins, DNA, purified subunits, peptides etc., targeting well-identified antigens. Changes in the nature of vaccine components and routes of administration are imposing a need for new adjuvants to be developed. There is also currently much interest in designing vaccines capable of eliciting strong cellular immune responses of the Th1 type. The latter encompasses both the induction of cytotoxic and Th1 helper cellular responses leading to the activation of immune effector mechanisms as well as the production of immunostimulatory cytokines such as interferon gamma. The induction of such Th1 responses is highly desirable for vaccines targeting either chronic viral diseases, infections linked to viral pathogens or cancer (therapeutic vaccines) (36, 37). In addition, induction by vaccines of local (e.g. mucosal) or polarized (i.e. Th1 or Th2) immune responses is needed in a number of circumstances. An additional requirement is that adjuvants should not elicit unacceptable local reactions, when used as part of prophylactic vaccines, as well as therapeutic vaccines. However, adjuvants, which reach these new expectations and might be useful in humans, have yet to be identified.

\subsection{New Developments in Adjuvants for Vaccines}

Adjuvants have been considered to be an essential component of most inactivated vaccines for the last five decades. Where live attenuated vaccines may trigger endogenous mechanisms that enhance immune responses, non-living materials do not generally exhibit this capability and may require the addition of exogenous agents to achieve appropriate levels of immunogenicity. In addition to stimulating immune responses, adjuvants can serve other purposes, such as improving the stability of vaccines containing more than one component or reducing the toxicity of vaccine constituents. Inorganic gels, consisting of aluminum or calcium phosphate, sulfate, oxide or hydroxide, in varying proportions, were among the first adjuvants and still are the only ones licensed for human use (36).

The development of recombinant methods for the production of proteins and improvements in large scale purification of proteins and polysaccharides, have allowed vaccine developers to move away from the use of whole killed bacteria and viruses and toward the use of purified subunits. Greater understanding of immune mechanisms has stimulated efforts to target vaccines to elicit specific mechanisms of resistance to bacterial as well as viral pathogens. Together these forces have driven a search for novel adjuvants with specific physical and immunological properties optimally suited for vaccines of particular diseases.

\section{Results}

\subsection{Problems in Development of Adjuvants}

Despite the development of many potent adjuvant formulations with vaccine antigen during the last 80 - 90 years, aluminum compounds are still the only approved adjuvants used for routine human vaccines. A major problem in the development of vaccine adjuvants for routine human vaccines is that rare adverse reactions, such as one in several thousand doses, may not be detected until late in the development program. Additionally, there are some other problems encountered during the development of adjuvants for human vaccines. These problems are mentioned below.

\subsubsection{Limited Adjuvanticity}

Several adjuvants act with certain specific antigens and are not effective with other antigens. For example, aluminum compounds did not exhibit an adjuvant effect when used with typhoid vaccine (38), influenza hemagglutinin antigen (39) and Haemophilus influenzae type b (Hib) capsular polysaccharide conjugated to tetanus toxoid (40). Model or standard antigens including ovalbumin (chicken egg white) and influenza hemagglutinin have been suggested to study the adjuvanticity of new formulations (40). These model antigens may be useful for initial preliminary screening of vaccine adjuvants, yet it is recommended for most of the development work to be done with the antigen for which the adjuvant is being developed. Ovalbumin, being a weak immunogen, may be a suitable antigen for evaluation of adjuvants, yet there are several problems with its use as a model or standard antigen. 1) Ovalbumin does not have any clinical significance. 2) Studies on animals using ovalbumin have employed very high doses of the protein (41) that are not clinically acceptable and may be maximal doses, and it would not be possible to discriminate small differences among adjuvant formulations. 3) No functional antibody assay can be performed, as ovalbumin does not have biological activity.

\subsubsection{Sub-Optimal Use of Aluminum Adjuvants}

Aluminum compounds used as vaccine adjuvants include aluminum phosphate, aluminum hydroxide and alum-precipitated vaccines. These have often been referred to as "alum". Aluminum hydroxide has been found to be a more potent adjuvant than aluminum phosphate (42). Aluminum hydroxide adjuvanted antigens showed similar or superior antibody responses than the antigens given with Freund's complete adjuvant (FCA) (43). Aluminum hydroxide is a good adjuvant for weak antigens 
Gupta A et al.

in mice yet saponin and FCA are more potent adjuvants than aluminum hydroxide for strong antigens (44).

Aluminum adjuvants have been described as difficult to manufacture in a physicochemically reproducible way. Adsorption of antigens on aluminum adjuvants depend upon physical and chemical characteristics of antigen, type of aluminum adjuvant and conditions of adsorption (13). The immunogenicity of antigens adsorbed onto aluminum adjuvants depend upon a number of factors, most importantly on the dose of the adjuvant. The dose of aluminum adjuvant affects the overall immunogenicity $(42,44)$. Although small amounts of aluminum adjuvant are required for complete adsorption of the antigen (low dose), Although small amounts of aluminum adjuvant are required for complete adsorption of the bacterial or viral antigens (low dose), even though they may not show an optimal adjuvant effect (42). As the amount of aluminum adjuvant is increased, the adjuvant effect rises to a certain concentration after which the adjuvant effect declines with further increases in aluminum adjuvant concentration (42). The reasons for this optimum concentration of adjuvant are unknown. We speculate that a minimum amount of aluminum compound is necessary to form a depot at the site of injection or to optimally stimulate macrophages. Excessive amounts of aluminum compounds may suppress immunity by covering the antigen completely with mineral compounds or by being toxic to macrophages, as aluminum compounds are somewhat cytotoxic to macrophages. Several studies showed lower immunogenicity/potency for aluminum adsorbed vaccines diluted in saline in mice than those diluted in the aluminum adjuvant (42).

\subsubsection{Animal Models}

There are no reliable animal models for many diseases against which vaccines are being developed. Therefore, it becomes very difficult to evaluate adjuvants for these vaccine antigens. Different animal species respond differently to various adjuvants. Even within one animal species, different strains, particularly mice, behave differently to various adjuvants. Variable response of different strains of mice to vaccine antigens has been known for years (45). Different adjuvants appeared to behave very differently in different strains of mice. For instance, $\gamma$-inulin, which has been shown to be a good adjuvant (46), did not show much adjuvanticity with diphtheria toxoid in CD-l outbred mice, whereas it was a good adjuvant in inbred Balb/C and C57 mice. However, this observation suggested that the results of animal studies, particularly when the study is performed on one strain of single species of animals, should be interpreted cautiously. Despite wide variations observed in different strains of mice with certain adjuvants, mice will still be used widely for evaluating adjuvant formulations due to the availability of reagents for subclass and cytokine analysis of mice. Additionally, mice are cheap and easy to handle compared to other animal models. In this study, we selected Balb/C mice, including responder and non-responder mice. Non-responding mice did not develop any anti-HBsAg antibody response $(<10 \mathrm{mIU} / \mathrm{mL})$, while responder mice showed detectable anti-HBsAg antibodies (>10 mIU/mL).

\subsubsection{Problems with Antibody Assays}

Antibody response of animals to antigens with various adjuvants is usually measured by immunoassays. In the recent years, the enzyme-linked immunosorbent assay (ELISA) has been widely used for measuring the concentrations of antibodies in human and animal sera due to its simplicity, ease of automation, availability of stable reagents and objective interpretation. A major problem with the ELISA method is that different groups of scientists assign antibody concentrations by different methods, including titers (reciprocal of the highest dilution of serum giving a specified absorbance), absorbance (optical density) units (usually multiplication of absorbance) and reciprocal of the dilution showing that absorbance, arbitrary units, international units or weight-based units $(\mu \mathrm{g} / \mathrm{mL})$.

Results of titers and absorbance units are not usually determined against an ELISA reference serum and these are affected by environmental factors such as temperature, time and humidity, particularly during the last step involving enzyme and substrate interaction. Results obtained on different days on the same samples in the same laboratory are sometimes difficult to reproduce. In certain cases, the ELISA reference serum is calibrated to international units, by a functional antibody assay against an international or national standard. In this case results can be compared among different laboratories, yet the differences in avidities of the ELISA reference serum and unknown sera cause discrepancies between ELISA titers and titers obtained by functional antibody assays (42). Nevertheless, none of these methods are suitable for assigning antibody concentrations for IgG subclasses. Results of IgG subclass antibody concentrations expressed in titers or absorbance units cannot be reliably compared, even from the same laboratory because sensitivity of various IgG subclasses of ELISA varies depending upon the quality of the antibody in the conjugates. Another commonly used method of assigning antibody concentrations in ELISA is by weight-based units $(\mu \mathrm{g} / \mathrm{mL})$ determined by capturing known concentrations of purified immunoglobulins on ELISA plates and comparing these against antibodies captured with antigen (47).

\subsection{Adjuvant Influences on the Immune Response to Soluble Protein Antigens}

Adjuvants function to modify the immune response on various levels. In general, their activity can be classified into four categories based on their mechanism of action as shown in Table 1. On the other hand, the effect of adjuvants can also be classified by outcome. For example, alum is more likely to generate an antibody 
response without DTH while the complex mixture of inactivated mycobacteria in oil (Freund's complete adjuvant) generates both DTH/cytotoxicity and antibody response. It has even been proposed that mixing adjuvants with different ratios of Th1 and Th2, may produce useful vaccine adjuvant formulations (48). While dramatic progress has been made in understanding the mechanisms involved in these adjuvant activities and in the design of new adjuvants, there is rarely a dramatic shift from one side of the response (antibody) to the other (cellular). Classically, the pivotal event in defining antibody vs. DTH/cytotoxicity is the activity of T helper cells (Th1 biasing towards DTH/cytotoxicity vs. Th2 biasing towards antibody). While that final event is important for the biasing of the response, modifying any of the multiple prior steps will act to enhance the overall response dramatically and perhaps influence the final pivotal Th1/Th2 events to varying degrees.

One observation that is difficult to explain and predict is that most adjuvants are often more active for some antigens than others (i.e. antigen-specific). For example, many antigens will yield equal antibody titers whether the adjuvant is alum or Freund's while others require Freund's based adjuvants for optimal antibody titer. The same is true for various T cell responses. For example, QS21 was quite effective for some HIV antigens but not very effective for others $(41,49)$.

Since many adjuvants function to induce inflammatory events and are proposed for use in prophylactic vaccines, defining their range of toxicity and/or reactogenicity as well as their adjuvant activity is required. If the potential side effects are known, an intelligent risk benefit analysis can be made. Such an analysis would take into account not only the target population (naive or infected; pediatric or adult) but also the severity of the disease (HIV vs. HCV). These risks might also change as new therapies for treatment of a disease develop (i.e. anti-virals for HIV) or new locations for outbreaks are identified. Typically, adjuvants are compared to the side effect profile of alum. Alum side effects include muscle soreness and occasional fever, depending on the antigen. Several adjuvants with surprising toxicities have been identified. For example, QS-21 (saponin, predominantly an antigen uptake enhancing adjuvant) caused profound splenomegaly in mice and red cell lysis in humans (41). This observation led additional studies to develop analogs that retained the adjuvant activity but had less of the toxicity.

In addition to acceptable toxicity/pyrogenicity, additional requirements are also placed on adjuvants. Adjuvants must form stable complexes (if necessary) with the various components of the vaccine and they should be non-immunogenic. In addition to generating a good initial immune response, they must be effective in generating a good anamnestic (or memory) response. Finally, in addition to generating strong and long lasting $\mathrm{T}$ cell immunity, they should not induce autoimmunity. The autoimmunity issue could be especially critical for adjuvants that activate both $\mathrm{T}$ and $\mathrm{B}$ cell responses via a general over stimulation of the immune response.

While the adjuvant underlying its focus on proteinbased, the underlying focus was on protein-based vaccines. Given the increased emphasis on the use of DNA vaccines, a few specific comments on the value of adjuvants in these settings are warranted. Many DNA vaccines, in fact, function mainly to generate a constant source of antigen at a local site and as such, many adjuvants effective for proteins would also be useful for DNA vaccines (50). The dilemma in this setting is that the time to production of protein after DNA injection may be delayed compared to the classic adjuvant use at the time of protein administration. Furthermore, DNA vaccines are inherently of value because they are readily engineered to contain multiple epitopes and should provide for continual synthesis of protein at the injection site for a longer period of time than injected protein.

Table 1. Classification of Adjuvants by Mechanism of Enhancement of the Immune System ${ }^{\text {a }}$

\begin{tabular}{|c|c|c|}
\hline Action & Example & Mechanism \\
\hline Antigen retention & $\begin{array}{l}\text { Alum, oil emulsions (montanide, MF 59), } \\
\text { non-ionic block copolymers }\end{array}$ & $\begin{array}{l}\text { APC have more time for antigen uptake and presentation; } \\
\text { newly recruited cells have access to antigen. }\end{array}$ \\
\hline Antigen uptake & ISCOMS, alum, liposomes, QS21 & $\begin{array}{l}\text { Enhanced uptake (perhaps due to facilitation of antigen } \\
\text { crossing the membrane) increases the number of present- } \\
\text { ing sites on APC. }\end{array}$ \\
\hline $\begin{array}{l}\text { Activation of innate } \\
\text { response }\end{array}$ & LPS, CpG, MPLA, MDT, CWS & $\begin{array}{l}\text { Activation of TLR (toll-like receptors) results in increase } \\
\text { in cytokine and chemokine production and ultimately } \\
\text { maturation of APC. }\end{array}$ \\
\hline Cytokine enhancement & IFN gamma, IL-1, IL-2 & $\begin{array}{c}\text { Increases in the number of T cells modulates the direction } \\
\text { of the response (Th1 vs. Th2). }\end{array}$ \\
\hline
\end{tabular}


Gupta A et al.

\subsection{Example of Plant-Based Adjuvant-QS21}

The vaccine adjuvant QS-21 is a highly purified saponin derived from the bark of the South American tree, Quillaja saponaria Molina (51). It is a water-soluble quillaic acidbased triterpene with a complex acylated 3, 28-O-bisglycoside structure (51, 52). Furthermore, QS-21 has been shown to be an effective immunological adjuvant with a wide variety of antigens and to have a relatively low toxicity in preclinical studies on mice. It enhances antibody responses, including IgG2a $(51,52)$ and antigen-specific interferongamma and IL-4 production (53). It also enhances CD8 + cytotoxic T lymphocytes in animal studies $(54,55)$.

QS-21 has been evaluated in a large number of vaccines in phase I and phase II human clinical trials (52). These vaccines include cancer immunotherapeutics $(56,57)$, HIV recombinant envelope (58) and malarial antigens (59). To date, QS-21 has been tested on more than 2600 individuals. In most of these studies, QS-21 doses of $50-100$ $\mu \mathrm{g}$ were utilized. All vaccines were given either by the intramuscular or subcutaneous route. QS-21 was shown to strongly enhance the responder rate and antibody titers to the ganglioside portion of the conjugated melanoma antigen GM2-KLH $(56,57)$. This vaccine is now in phase III of clinical trials. QS-21 was also a critical component of the adjuvant formulation of the first malaria recombinant antigen vaccine, to protect the majority of vaccinated volunteers against parasite challenge (59). Hence, it appears to be a promising adjuvant for human vaccines. Vaccines containing QS-21 have generally been well tolerated. Systemic side-effects are infrequent. Local reactions, typically consisting of transient mild or moderate pain, tenderness and induration have been seen in most studies. However, a more marked local reaction, consisting of severe immediate injection pain was observed in some individuals in two studies. Immediate injection pain, categorized as severe and a burning sensation was reported by $12 \%$ of individuals receiving intramuscularly administered QS-21 in an HIV-1 gp120 vaccine trial (57-59). QS-21 however, is a surfactant with lytic effect on cells $(51,52)$; a factor that may be associated with immediate injection site pain. In addition, formulation characteristics such as buffer, $\mathrm{pH}$ and excipients are known to affect immediate injection pain to some pharmaceuticals. Various strategies may reduce or eliminate injection-associated pain. These strategies include a change of injection route or site, $\mathrm{pH}$, distraction of recipient during immunization, formulation with an excipient that forms an emulsion, complex or mixed micelle with the pharmaceutical (60) or inclusion of a local anesthetic such as lidocaine or procaine (60). Thus, it hardly needs to be emphasized that the development of more plant-based adjuvants are highly desirable for various diseases. In addition, the number of adjuvant candidates isolated or purified from various medicinal plants e.g. Picrorhiza kurroa (61), Boswellia serrata (62), Emblica officinalis (19) etc., showed remarkable activity against a number of vaccine antigens.

\section{Conclusions}

\subsection{Future Prospects}

Despite the problems encountered in the development of adjuvants for human vaccines, much progress has been made in the recent years, particularly in understanding the molecular basis of action of adjuvants and different types of cells ( $B, T$, natural killer and macrophages) involved in immune response and the correlations of immunity to various diseases. This led to the development of adjuvants, which can selectively modulate immune response and even adjuvants, which can selectively elicit T-cells. The basic knowledge of adjuvant action is very important for developing suitable vaccines for newly emerging diseases such as AIDS or for diseases against which no effective vaccines are available. It is likely that aluminum compounds will continue to be used with current human vaccines for many years due to their excellent track record of safety and adjuvanticity. For infections (viral or bacterial) that can be prevented or reduced by induction of serum antibodies (IgG and its isotypes), aluminum adjuvants formulated under optimal conditions are the adjuvants of choice. However, for purified, subunit or synthetic vaccines and combination vaccines comprised of purified vaccine antigens, more potent adjuvants may be necessary. Due to limitations of aluminum adjuvants, especially their inability to elicit cell-mediated immune responses, such as cytotoxic T-cell responses, there is a need for alternative vaccine adjuvants against specific antigens, particularly for bacterial or viral diseases in which cell mediated immune responses are important for prevention or cure. Based on pre-clinical and preliminary clinical observations, it appears that the range of adjuvants accepted for human vaccines will expand in the coming years.

\section{References}

1. Glenny AT, Pope CG, Waddington H, Wallace U. Immunological notes. XVII-XXIV. J Pathol Bacteriol. 1926;29(1):31-40.

2. Lewis PA, Loomis D. Allergic Irritability : The Formation of AntiSheep Hemolytic Amboceptor in the Normal and Tuberculous Guinea Pig. J Exp Med.1924;40(4):503-15.

3. Freund J, Casals J, Hosmer EP. Sensitization and Antibody Formation after Injection of Tubercle Bacilli and Paraffin Oil. Exp Biol Med.1937;37(3):509-13.

4. Johnson AG, Gaines S, Landy M. Studies on the O antigen of Salmonella typhosa. V. Enhancement of antibody response to protein antigens by the purified lipopolysaccharide. J Exp Med. 1956;103(2):225-46.

5. Ellouz F, Adam A, Ciorbaru R, Lederer E. Minimal structural requirements for adjuvant activity of bacterial peptidoglycan derivatives. Biochem Biophys Res Commun. 1974;59(4):1317-25.

6. Baker PJ, Hiernaux JR, Fauntleroy MB, Stashak PW, Prescott B Cantrell JL, et al. Ability of monophosphoryl lipid A to augment the antibody response of young mice. Infect Immun. 1988;56(12):3064-6.

7. Mbawuike IN, Wyde PR, Anderson PM. Enhancement of the protective efficacy of inactivated influenza A virus vaccine in aged mice by IL-2 liposomes. Vaccine. 1990;8(4):347-52.

8. Hibberd PL, Rubin RH. Immunization strategies for the immuno- 
compromised host: the need for immunoadjuvants. Ann Intern Med.1989;110(12):955-6.

9. Grun JL, Maurer PH. Different T helper cell subsets elicited in mice utilizing two different adjuvant vehicles: the role of endogenous interleukin 1 in proliferative responses. Cell Immunol. 1989;121(1):134-45.

10. Vogel FR, Powell MF. A compendium of vaccine adjuvants and excipients. Pharm Biotechnol.1995;6:141-228.

11. Abraham E. Intranasal immunization with bacterial polysaccharide containing liposomes enhances antigen-specific pulmonary secretory antibody response. Vaccine.1992;10(7):461-8.

12. Guzman CA, Molinari G, Fountain MW, Rohde M, Timmis KN, Walker MJ. Antibody responses in the serum and respiratory tract of mice following oral vaccination with liposomes coated with filamentous hemagglutinin and pertussis toxoid. Infect Immun. 1993;61(2):573-9.

13. Edelman R. Vaccine adjuvants. Rev Infect Dis. 1980;2(3):370-83.

14. Vogel FR. Improving vaccine performance with adjuvants. Clin Infect Dis. 2000;30 Suppl 3:S266-70.

15. Gupta A, Khamkar PR, Chaphalkar SR. Immunomodulatory and anti-inflammatory activity of aqueous extract of leaf, stem and root of Ficus religiosa on human whole blood and peripheral blood mononuclear cells. Int J Med Pharm Res. 2014;2(4):732-9.

16. Gupta A, Khajuria A, Singh J, Bedi KL, Satti NK, Dutt P, et al. Immunomodulatory activity of biopolymeric fraction RLJ-NE-205 from Picrorhiza kurroa. Int Immunopharmacol. 2006;6(10):1543-9.

17. Khajuria A, Gupta A, Suden P, Singh S, Malik F, Singh J, et al. Immunomodulatory activity of biopolymeric fraction BOS 2000 from Boswellia serrata. Phytother Res. 2008;22(3):340-8.

18. Agarwal R, Diwanay S, Patki P, Patwardhan B. Studies on immunomodulatory activity of Withania somnifera (Ashwagandha) extracts in experimental immune inflammation.J Ethnopharmacol.1999;67(1):27-35.

19. Gupta A, Chaphalkar SR. Use of flow cytometry to measure the immunostimulatory activity of aqueous extract of Jasminum auriculatum. Int J Current Adv Res. 2015.

20. Ziauddin M, Phansalkar N, Patki P, Diwanay S, Patwardhan B. Studies on the immunomodulatory effects of Ashwagandha. J Ethnopharmacol.1996;50(2):69-76.

21. Patwardhan B, Kalbag D, Patki PS, Nagasampagi BA. Search of immunomodulatory agents--A review. Indian Drugs. 1990;28:56-63.

22. Cherwinski HM, Schumacher JH, Brown KD, Mosmann TR. Two types of mouse helper T cell clone. III. Further differences in lymphokine synthesis between Th1 and Th2 clones revealed by RNA hybridization, functionally monospecific bioassays, and monoclonal antibodies. J Experimental Med.1987;166(5):1229-44.

23. Mosmann TR, Sad S. The expanding universe of T-cell subsets: Th1, Th2 and more. Immunol Today. 1996;17(3):138-46.

24. Romagnani S. Human TH1 and TH2 subsets: doubt no more. Immunol Today. 1991;12(8):256-7.

25. Brewer JM, Richmond J, Alexander J. The demonstration of an essential role for macrophages in the in vivo generation of IgG2a antibodies. Clin Exp Immunol. 1994;97(1):164-71.

26. Lanzavecchia A. Receptor-mediated antigen uptake and its effect on antigen presentation to class II-restricted T lymphocytes. Annu Rev Immunol.1990;8:773-93.

27. Neefjes JJ, Momburg F. Cell biology of antigen presentation. Curr Opin Immunol. 1993;5(1):27-34.

28. Cambier JC, Pleiman CM, Clark MR. Signal transduction by the B cell antigen receptor and its coreceptors. Annu Rev Immunol. 1994;12:457-86.

29. Caux C, Liu YJ, Banchereau J. Recent advances in the study of dendritic cells and follicular dendritic cells. Immunol Today. 1995;16(1):2-4.

30. Driscoll J.The role of the proteasome in cellular protein degradation. Histol Histopathol. 1994;9(1):197-202.

31. Abel G, Szollosi J, Chihara G, Fachet J. Effect of lentinan and mannan on phagocytosis of fluorescent latex microbeads by mouse peritoneal macrophages: a flow cytometric study. Int J Immunopharmacol.1989;11(6):615-21.

32. Jiang W, Swiggard WJ, Heufler C, Peng M, Mirza A, Steinman RM et al. The receptor DEC-205 expressed by dendritic cells and thy- mic epithelial cells is involved in antigen processing. Nature. 1995;375(6527):151-5.

33. Ribi HO, Ludwig DS, Mercer KL, Schoolnik GK, Kornberg RD. Three-dimensional structure of cholera toxin penetrating a lipid membrane. Science. 1988;239(4845):1272-6.

34. Chinnah AD, Baig MA, Tizard IR, Kemp MC. Antigen dependent adjuvant activity of a polydispersed beta-(1,4)-linked acetylated mannan (acemannan). Vaccine.1992;10(8):551-7.

35. Allison AC, Byars NE. Immunological adjuvants and their mode of action. Biotechnology. 1992;20:431-49.

36. Shirodkar S, Hutchinson RL, Perry DL, White JL, Hem SL. Aluminum compounds used as adjuvants in vaccines. Pharm Res. 1990;7(12):1282-8.

37. Al-Shakhshir RH, Regnier FE, White JL, Hem SL. Contribution of electrostatic and hydrophobic interactions to the adsorption of proteins by aluminium-containing adjuvants. Vaccine. 1995;13(1):41-4.

38. Cvjetanovic B, Uemura K. The Present Status of Field and Laboratory Studies of Typhoid and Paratyphoid Vaccines with Special Reference to Studies Sponsored by World Health Organization. Bull World Health Organ. 1965;32:29-36.

39. Davenport FM, Hennessy AV, Askin FB. Lack of adjuvant effect of A1PO4 on purified influenza virus hemagglutinins in man. J Immunol.1968;100(5):1139-40.

40. Chedid L. Adjuvants of immunity. Ann Inst Pasteur Immunol. 1985;136D(3):283-91.

41. O'Hagan DT, McGee JP, Holmgren J, Mowat AM, Donachie AM. Biodegradable microparticles as controlled release antigen delivery systems. Vaccine .1993;11:149-54.

42. Gupta RK, Rost BE, Relyveld E, Siber GR. Adjuvant properties of aluminum and calcium compounds. Pharm Biotechnol. 1995;6:229-48

43. Woodard LF. Adjuvant activity of water-insoluble surfactants. Lab Anim Sci.1989;39(3):222-5.

44. Bomford R. Aluminium salts: perspectives in their use as adjuvant. In: Gregoriadis G, Allison AC, Poste. G, editors. Immunological adjuvants and Vaccines. NATO ASI Series A: Life Sciences Vol. 179. Proceedinas of a NATO Advanced Study institute on Immunological Aldjuvants and Vaccines, 24 June - 5 July 1988, Cape Sounion Beach, Greece. 1989. pp. 35-41.

45. Matsuhasi T. Proceedings of an Informal Consultation on the World Health Organization Requirements for Diphtheria, Tetanus, Pertussis and Combined Vaccines. 2 ed. Department of Health and Human Services United States Public Health Service Bethesda; 1991.pp.

46. Cooper PD. The selective induction of different immune responses by vaccine adjuvants. In: Ada GL editor. Strategies in Vaccine Design Landes RG Company Austin .; 1994.

47. Zollinger WD, Boslego JW. A general approach to standardization of the solid-phase radioimmunoassay for quantitation of classspecific antibodies. J Immunol Methods. 1981;46(2):129-40.

48. Moingeon P, Haensler J, Lindberg A. Towards the rational design of Th1 adjuvants. Vaccine. 2001;19(31):4363-72.

49. Schijns VE. Immunological concepts of vaccine adjuvant activity Curr Opin Immunol. 2000;12(4):456-63.

50. Spack EG, Sorgi FL. Developing non-viral DNA delivery systems for cancer and infectious disease. Drug Discov Today. 2001;6(4):186-97.

51. Kensil CR. Saponins as vaccine adjuvants. Critical Reviews ${ }^{\mathrm{TM}}$ in Therapeutic Drug Carrier Systems. 2 ed; 1996. pp.1-55.

52. Kensil CR, Kammer R. QS-21: a water-soluble triterpene glycoside adjuvant. Expert Opin Investig Drugs. 1998;7(9):1475-82.

53. Kim SK, Ragupathi G, Musselli C, Choi SJ, Park YS, Livingston PO. Comparison of the effect of different immunological ad juvants on the antibody and T-cell response to immunization with MUC1-KLH and GD3-KLH conjugate cancer vaccines. Vaccine. 1999;18(7-8):597-603.

54. Hancock G. Formulation of the purified fusion protein of respiratory syncytial virus with the saponin QS-21 induces protective immune responses in Balb/c mice that are similar to those generated by experimental infection. Vaccine. 1995;13(4):391-400.

55. Newman MJ, Wu JY, Gardner BH, Munroe KJ, Leombruno D, 
Recchia J, et al. Saponin adjuvant induction of ovalbuminspecific CD8+ cytotoxic $\mathrm{T}$ lymphocyte responses. J Immunol. 1992;148(8):2357-62.

56. Helling F, Zhang S, Shang A, Adluri S, Calves M, Koganty R, et al. GM2-KLH conjugate vaccine: increased immunogenicity in melanoma patients after administration with immunological adjuvant QS-21. Cancer Res. 1995;55(13):2783-8.

57. Livingston PO, Adluri S, Helling F, Yao TJ, Kensil CR, Newman MJ, et al. Phase 1 trial of immunological adjuvant QS-21 with a GM2 ganglioside-keyhole limpet haemocyanin conjugate vaccine in patients with malignant melanoma. Vaccine. 1994;12(14):1275-80.

58. Keefer MC, Wolff M, Gorse GJ, Graham BS, Corey L, ClementsMann ML, et al. Safety profile of phase I and II preventive HIV type 1 envelope vaccination: experience of the NIAID AIDS Vaccine Evaluation Group. AIDS Res Hum Retroviruses. 1997;13(14):1163-77.

59. Stoute JA, Slaoui M, Heppner DG, Momin P, Kester KE, Desmons
P, et al. A preliminary evaluation of a recombinant circumsporozoite protein vaccine against Plasmodium falciparum malaria. RTS,S Malaria Vaccine Evaluation Group. $N$ Engl J Med. 1997;336(2):86-91.

60. Brazeau GA, Fung HL. An in vitro model to evaluate muscle damage following intramuscular injections. Pharm Res. 1989;6(2):167-70.

61. Khajuria A, Gupta A, Singh S, Malik F, Singh J, Suri KA, et al. RLJ-NE-299A: a new plant based vaccine adjuvant. Vaccine. 2007;25(14):2706-15.

62. Khajuria A, Gupta A, Malik F, Singh S, Singh J, Gupta BD, et al. A new vaccine adjuvant (BOS 2000) a potent enhancer mixed Th1/ Th2 immune responses in mice immunized with HBsAg. Vaccine. 2007;25(23):4586-94. 